\title{
Effect of cawthorne and cooksey exercises on balance and quality of life of 60 to 80 year- old individuals in Shiraz: A randomized clinical trial
}

\author{
Abolghasem Fallahzadeh Abarghuei ${ }^{1}$, Mahsa Fadavi-Ghaffari*1, Safoura Tousi ${ }^{2}$, Malek Amini ${ }^{1}$, Ali Reza Salehi \\ Received: 24 Nov 2017 \\ Published: 19 Aug 2018
}

\section{Abstract}

Background: One of the vulnerable populations of any community are the elderly, who are exposed to poor balance, falls, injuries, reduced quality of life (QoL) and independency, and early death. Considering the importance of balance in independently performing activities of daily living (ADL) and its impact on QoL, in this study, we aimed at investigating the effect of Cawthorne and Cooksey exercises (CCE) on QoL and balance of 60 to 80 year-old individuals in Shiraz, Iran.

Methods: This clinical trial study was conducted on forty 80 to 60 -year-old individuals of Jahandideghan retirement center in Shiraz (20 in the intervention and 20 in the control groups) in 2014. The intervention group did CCE in three 60- minute sessions a week for 2 months. The balance status of the participants was assessed with Berg balance test, and their QoL was also examined using SF36 Quality of Life Questionnaire before the intervention and at 2 months post intervention. Data were analyzed by independent $t$ test in SPSS software Version 19.

Results: The results of the study revealed that the difference was significant in the balance change scores between the 2 groups $(\mathrm{p}<0.001$, effect size $=0.78)$. Change differences in the QoL scores were significant between the 2 groups as well $(p<0.001$, effect size $=0.39$ ).

Conclusion: CCT improved QoL and balance of 60 to 80 year- old Shirazians, so considering the low cost, easy implementation, and considerable impact of the exercises, CCT could be used to prevent and treat balance disorders of the elderly.

Keywords: Balance, Cawthorne and Cooksey exercises, Quality of life, The elderly

Copyright $\odot$ Iran University of Medical Sciences

Cite this article as: Fallahzadeh Abarghuei A, Fadavi-Ghaffari M, Tousi S, Amini M, Salehi AR. Effect of cawthorne and cooksey exercises on balance and quality of life of 60 to 80 year- old individuals in Shiraz: A randomized clinical trial. Med J Islam Repub Iran. 2018 (19 Aug);32:74. https://doi.org/10.14196/mjiri.32.74

\section{Introduction}

Aging is a process through which slow, spontaneous, and progressive changes occur in the body structure over time and affect human life (1-3). In recent years, with increase in life expectancy, medical technology improvement, public health promotion, and mortality reduction, the life span of our population has increased (4). Thus, it is expected that the elderly population increase in the future. Currently, the number of worldwide elder population is 600 million, and it is predicted to reach 2 billion by 2050 , which would be $22 \%$ of the world's population at that time $(5,6)$. Iran's population is aging along with the world's population. Already, $7 \%$ of the population in Iran

\footnotetext{
Corresponding author: Mahsa Fadavi-Ghaffari, MahsafadaviMSC93@gmail.com

1. Department of Occupational Therapy, School of Rehabilitation Sciences, Iran University of Medical Sciences, Tehran, Iran.

2. School of Rehabilitation Sciences, Shiraz University of Medical Sciences, Shiraz, Iran.

3. Shiraz University of Medical Sciences, Shiraz, Iran.
}

are old and this number will double within the next 20 years, moreover, most of Iran's population will be old in the next 30 to 40 years (4-7). Common problems and diseases of the elderly include high blood pressure, urinary tract disorders, stroke, Parkinson's disease, Alzheimer's disease, dizziness, balance disorders, and falls (1).

Among the health problems of the elderly, functional independence problems are more important. Balance and walking are considered as indicators of elders' functional independence. Balance is defined as the ability to maintain a condition for voluntary activities and coping with protuberances (internal or external), and in biomechanical

$\uparrow$ What is “already known” in this topic:

This randomized clinical trial was the first to examine the effect of Cawthorne and Cooksey exercises on balance and quality of life of 60 to 80 year- olds in Shiraz.

$\rightarrow$ What this article adds:

CCT improved balance and QoL scores. The difference in balance change scores and change differences in the QoL scores were significant between the 2 groups $(\mathrm{p}<0.001$, effect size $=$ $0.78,0.39)$. 
aspect, maintaining body of mass (BOM) in base of support $(\mathrm{BOS})$ area $(8,9)$. Maintaining balance requires an interaction between sensory (visual, vestibular, somatosensory) and motor systems within the central nervous system (10). With the onset of aging, metabolic and physiological system changes which may affect musculoskeletal, vestibular, somatosensory, and visual systems and the ability of elders to maintain balance, putting them at risk of injuries, such as falls, fractures, and long disability. These negative changes threat QoL of the elderly and prevent them to perform their activities of daily livings (ADLS) (11).

QoL has become one of the most important contemporary issues in health care. One of the major goals established in "Healthy People 2010" is to increase quality and health of life. During the later years, QoL has been found to be as important as duration of life for many older individuals; however, it is currently unclear whether physical activity plays a role in improving QoL in older adults. Only a limited amount of research has been conducted in this area, and results from this research are equivocal (12).

Decline of balance in the elderly would increase their risk of fall. As falls can affect performance and independence, identifying those at risk of falls and developing diagnostic and treatment methods can improve QoL and independence, and can also reduce health care costs. Vestibular exercises, such as the ones described by Cawthorne and Cooksey, may serve as support for new arrangements of peripheral sensorial information, allowing new vestibular stimulation patterns necessary for new experiences to become automatic. CCE is the most common protocol for vestibular rehabilitation program, which involves balance centers, such as visual, proprioceptive, and vestibular activities; also, CCE can be used to enhance the formation of new environmental sensory information and it has the ability to improve balance responses, and thus, reduce falls $(13,14)$.

Rasteh et al. in 2009 found that CCE improves balance in the elderly (15). Also, the study results of Ribeiro et al. in 2005 showed that CCE improves balance and reduce falls in elderly women (14). Balance is a necessary component for performing activities of daily living and independent and dynamic living, so reduction in such abilities due to poor balance leads to dissatisfaction and reduction in QoL. Thus, it seems that by performing such exercises, the balance and QoL of elders improve. Therefore, we conducted this study to investigate the effect of CCE on balance and QoL of 60 to 80 year- old Shirazians.

\section{Methods}

This randomized clinical trial was approved by Ethics Committee of Shiraz University of Medical Sciences (reference number: CT-P-9361-6731) (IRCT registration number: IRCT2014061618111N1). The research sample consisted of forty 60 to 80 year- old Shirazians, who attended Shiraz Jahandideghan retirement center. The study was conducted during March and June 2014 in Shiraz Jahandidegan retirement center. In the present study, participants were selected with simple and non-probability method and were randomly allocated (simple randomizing method) into intervention $(n=20)$ and control $(n=20)$ groups. The first person was placed in each group by drawing lots and the next person was put in the other group; this procedure was continued until sample saturation. The intervention and control groups were matched by age, weight, and height. All samples were selected and were asked to provide a written informed consent form.

In this study, inclusion criteria were as follow: the MMSE questionnaire score of higher than 21; and no history of psychiatric, neuromuscular, orthopedic, neurological, and rheumatologic disorder. Exclusion criteria were participants' unwillingness to continue the study, and incidence of diseases such as stroke during the study.

\section{Procedure}

Participants completed a demographic form. Then, balance and QoL were assessed using Berg Balance Scale Questionnaire and SF36 Questionnaire, respectively, before the intervention. The intervention group performed CCE in 60- minute sessions 3 days a week for 2 months. All assessments were repeated for the 2 groups after 2 months. Also, the participants in the control group received rote exercises, such as music therapy, exercise therapy, and reminiscence.

\section{CCE included the following items:}

A) Eye and head movement, first sitting down slowly and then faster: (1) looking up and down, (2) looking to the right and left, (3) bringing the fingers closer and farther and looking at them, (4) moving the head slowly and then faster to the right and to the left, with eyes open, (5) moving the head slowly and then faster up and down, with eyes open, and (6) repeating steps 4 and 5 with eyes closed; (B) Head and body movement, sitting down: (1) placing an object on the floor, pick it up and bring it above the head and place it on the floor again, looking at the object the whole time, (2) shrinking the shoulders and make circular movements, (3) bending forward and taking an object through the back and front of the knees; (C) Standing up exercises: (1) repeating A and B2, (2) siting down and standing up twice, (1) siting down and standing up twice, with eyes closed, (2) standing up but turning to the right while standing, (3) standing up, but turning to the left while standing, (4) throwing a small ball from one hand to the other (above the horizon level), and (5) throwing a small ball from one hand to the other under the knees and vice versa. Other activities to improve balance are as follow: (1) climbing up and down the stairs using handrail, if necessary, (2) standing up and taking sudden 90o turns first with eyes open and then with eyes closed, (3) while walking, looking to the right and the left as if reading labels in the market, (4) practicing standing on one foot with the right foot and then the left foot first with eyes open and then with closed eyes, (5) standing up on a soft surface, (a) walking on the surface to get used to it, (b) walking on the tip of the feet first with eyes open and the, with eyes closed, (c) practicing exercise 4 on a soft surface, (6) circling around a person that is on the center who throws a large ball, which should be thrown back, and (7) walking around the room with eyes closed (14, 
16).

\section{Outcome measurement}

Berg Balance Scale: Berg Balance Scale (BBS) is a performance-based instrument that was originally developed by Berg to assess functional balance in older adults. BBS contains 14 items, each grading on a 5-point Likert scale (0 to 4), representing different levels of difficulty. The BBS total score ranges from 0 to 56, with higher scores indicating a higher level of functional balance. Acceptable levels of intra- (ICC: 0.95) and inter-rater (ICC: $0.93)$ reliability, with a moderate internal consistency (Cronbach's alpha $=0.62)$ and high validity, were demonstrated for the Persian version of BBS (17).

SF36 quality of life questionnaire: SF-36 is a questionnaire used to assess quality of life. The SF-36 consists of 2 main components (i.e. physical and mental) and 8 subscales, with scores ranging from 0 to 100 , with higher scores indicating better QoL (18). The Persian version of the SF-36 is a reliable and valid measure of health related QoL among the general population. The internal consistency showed that all 8 SF-36 scales met the minimum reliability standard, with the Cronbach's alpha coefficients, ranging from 0.77 to 0.90 . Also, convergent validity showed satisfactory results, ranging from 0.58 to 0.95 (19).

In the present study, BBS and SF36 were performed daily in Jahandideghan retirement center before and after the intervention for both control and intervention groups.

\section{Statistical analysis}

SPSS software Version 19 was used for data analysis. For descriptive analysis, we calculated the mean and standard deviation. Independent $t$ test was calculated to analyze change scores between the 2 groups. Significance level was set at 0.05 .

\section{Results}

A total of 40 elderly individuals participated in this study in 2 groups (control and intervention) (Fig. 1). As demonstrated in Table 1 , the mean of age, height, and weight of the participants in the intervention and control groups were in the same range. Intervention and control groups were matched by age, height, and weight.

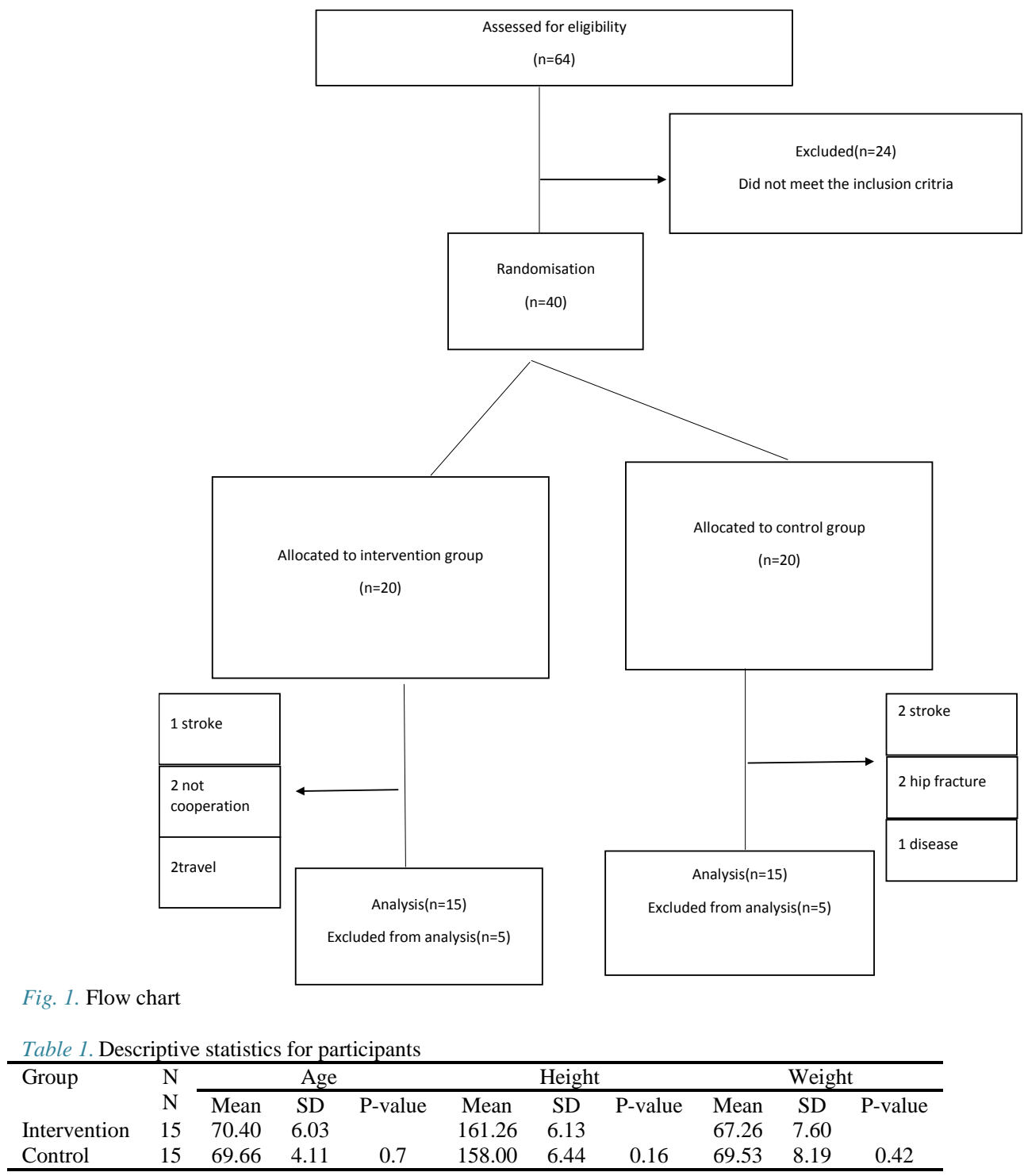


The effect of Cawthorne and Cooksey exercises on balance and quality of life of the elderly

Table 2. Comparison of change scores of Berg balance test between the 2 groups (control \& intervention) after the 2- month intervention

\begin{tabular}{|c|c|c|c|c|c|c|c|}
\hline & $\mathrm{N}$ & Mean of changes & Standard deviation & Mean difference & P-value & Confidence Interval 95\% & Effect Size \\
\hline Group & & & & & & Lower limit $\quad$ Upper limit & \\
\hline Intervention & $\begin{array}{l}15 \\
15\end{array}$ & -2.92 & 1.18 & 3.05 & 0.0001 & 3.71 & 0.78 \\
\hline
\end{tabular}

Table 3. The comparison of change scores of SF36 quality of life questionnaire between the 2 groups (control \& intervention) after the 2- month intervention

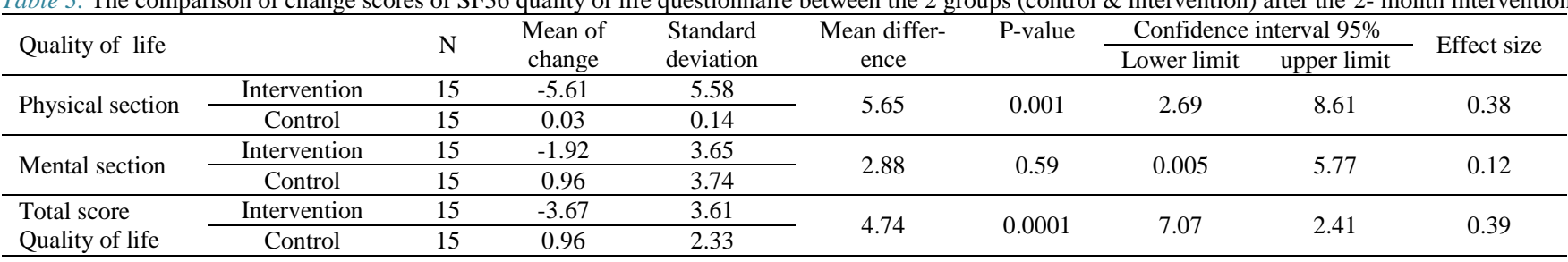

Based on Table 2, there were significant differences between the 2 groups in change scores of Berg's Balance Scale test $(\mathrm{p}<0.001)$.

Based on Table 3, the differences in change scores was significant in the physical part of SF36 Questionnaire. However, the change differences were not significant in the physiological part of SF36 Quality of Life Questionnaire. The mean changes of BBS and SF36 scores are demonstrated in Graphs 1 and 2.

Some participants were excluded from the study in the

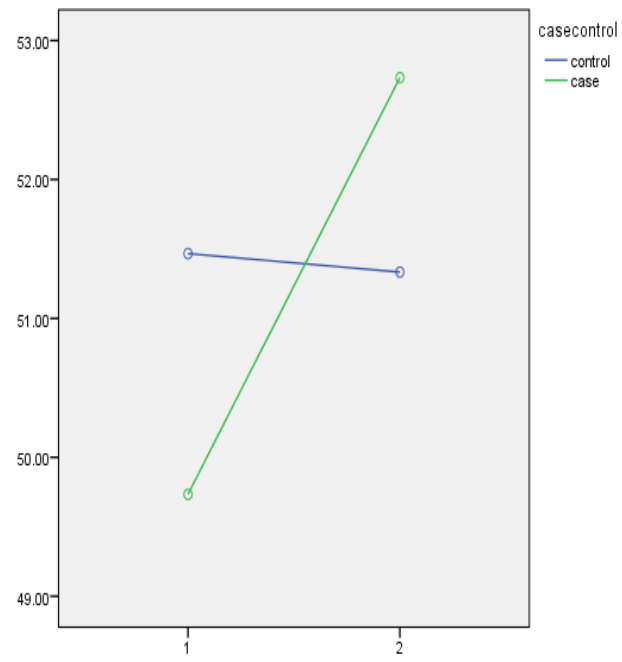

Graph 1. Mean changes of BBS scores

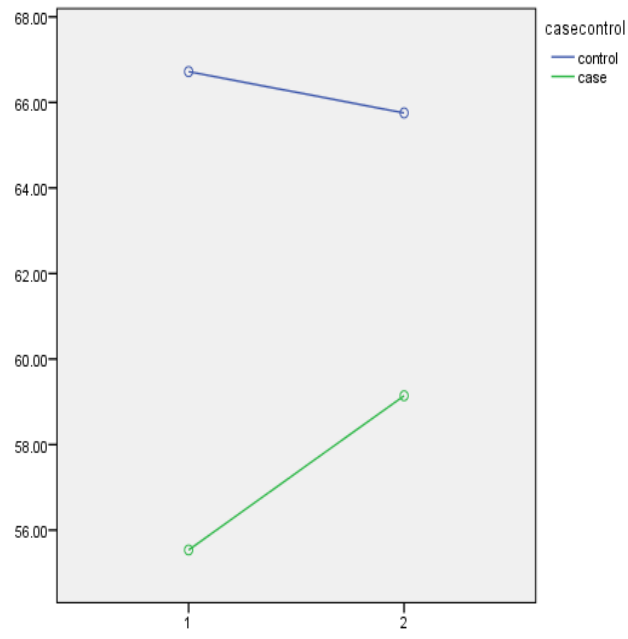

Graph 2. Mean changes of SF36 scores intervention and control groups due to the following reasons: 3 were excluded because of having a mild cerebral stroke, 1 participant had shingles disease, 2 were unwilling to continue the study, 2 went abroad, and 2 had an accident and hip fracture.

\section{Discussion}

The results revealed that CCE improved balance and QoL of the elderly participants, which is in line with previous studies $(14,15)$. However, to our knowledge, no study has assessed QoL and balance at the same time. In addition, the number of randomized clinical trial studies that evaluated the effect of CCE on the elderly was inadequate. Only few studies were done on healthy elderly such as Rybrol study in 2005, whose result was in line with that of ours (14).

In a review study conducted by Natalia in 2010 (16), 4 studies used CCE and expressed positive results for the influence of vestibular rehabilitation on postural control, administrative capacity, and QoL in the elderly and the middle-aged. However, the intervention time was inconsistent for the effect of CCE on balance and QoL. Duration of the intervention in Rasteh study was 3 times a week for 3 months; however, according to the results, 2 months is enough to improve balance in the elderly and prove the hypothesis of this study. Thus, intervention time in the present study was 2 months (performing the exercises 3 days a week). The results of the study showed that this 2- month intervention was enough to improve balance and QoL (physical section) (15). According to the results, QoL scores in physical dimension improved, but there was no significant effect in the psychological dimension, which may be due to insufficient time for the intervention. Balance improvement had positive effects on the physical aspects of the participants' QoL in 2 months, however, more time is needed for positive effects on the psychological aspect of Qol. It is suggested that during the intervention, effective interventions such as music, relaxation techniques, and breathing exercises be considered to improve the psychological aspect. Moreover, it should be determined whether these exercises, along with CCE, can promote psychological health in a 2- month intervention. According to the obtained results, CCE has a significant effect on balance and QoL of the elderly. Considering the possibility of easy implementation, low cost, ability to perform CCE in groups, and the fact that imbalance as a 
result of falls in many cases resulted in loss of independence and QoL, it is highly recommended to provide training and implement these exercises to elders.

\section{Conclusion}

CCE had a positive effect on QoL and balance of 60 to 80 year- old Shirazians. Thus, bearing in mind the low cost, easy implementation, and considerable impact of these exercises, they could be used to prevent and treat balance disorders in the elderly.

\section{Limitations}

The limitations in this study were small sample size, lack of blindness, and lack of follow-up.

\section{Acknowledgements}

This article was supported by Shiraz University of Medical Sciences. The authors declare that they had no conflict of interest.

\section{Conflict of Interests}

The authors declare that they have no competing interests.

\section{References}

1. Akbarfahimi N, Hossieni A, Jalili N. health promotion exercise for geriatrics. University of Social Welfare and Rehabilitation Sciences, 1st ed. 2010. pp.7-26.

2. Gregg E, Pereira M, Casperson C. Physical activity falls and fractures among older adults. J Am Geriatric Soc. 2000;48:883-93.

3. Clemson L, Fiatarone M, Bundy A, Cumming R, Weissel E, Munro $\mathrm{J}$. Integration of balance and strength training into daily life activity to reduce rate of falls in older people (the LiFE study): randomized parallel trial. BMJ. 2012;345:e4547.

4. Glogoski C, Chan M .Special needs of the older adult. Pedretti's occupational therapy. Mosby, 2005. pp.1171-11.

5. World Health Organization. About aging [cited Feb 02 2013]. Available at: URL: http://www.who.int/ageing/en

6. Statistical Center of Iran. Population Survey Results [cited Sep 13 2014]. Available at: URL: http://www.amar.org.ir/Default.aspx? tabid $=1160$

7. Hill A. Counselling older people: information for practitioners and policy makers. 2009; Available online from http://www.bacp.co.uk/research/information,sheets/R3.php

8. Shumway-Cook A, Woollcott MH. Motor Control: Theory and Practical Applications. 2nd ed; Baltimore; 2000.

9. Brownstein B, Bronner S. Functional movements in Orthopedic and Sports physical therapy: evaluation, treatment \& outcome. 1st ed. New York: Churchill Livingstone; 1997.

10. Radebold A, Cholewicki J, Polzhofer GK, Greene HS. Impaired postural control of the lumbar spine is associated with delayed muscle responses times in patients with chronic idiopathic low back pain. Spine. 2000;26(7):724-730.

11. Khalaji H, khajavi D. Human motor development: an approach to life .1st ed, Arak. 2005:711-768

12. Kotlyn AF. The association between physical activity and quality of life in older woman's health issue. J Woman Health Iss. 2001;11:471478 .

13. Shumway-cook A, Woollcott M. Motor control. 3td ed. New York: Lippincott Williams \& Wilkin; 2007.157-257.

14. Ribeiro A, Pereira J. Balance improvement and reduction of likelihood of falls in older women after Cawthorne and Cooksey exercises. Rev Bras Otorrinolaringol. 2005;71:38-46.

15. Rasteh H, Olyaei GR, Abdolvahab M, Jalili M, Jalaei SH. Efficacy of Cawthorne \& Cooksey exercise on balance improvement in elderly persons in Mashhad. Modern Rehabil. 2009;3(1):48-53.

16. Natalia A, Mayra C, Aratani F, Camila M, Heloísa H. systematic review about the effects of the vestibular rehabilitation in middle-age and A older adults. Rev Bras Fisioter. 2010;14(5):361-71

17. Salavati M, Negahban H, Mazaheri M, Soleimanifar M, Hadadi L, Hassan - Zahraee M. The Persian version of the Berg Balance Scale: Inter and intra - rater reliability and construct validity in elderly adults. Disabil Rehabil. 2012;34(20):1695-8.

18. Motamed N, Ayatollahi A, Zare N, Sadeghi Hassanabadi A. Validity and reliability of the Persian translation of the SF- 36 version 2 questionnaire. East Mediterr Health J. 2005 May;11(3):349-57.

19. Montazeri A, Goshtasebi A, Vahdaninia M, Gandek B. The Short Form Health Survey (SF-36): Translation and validation study of the Iranian version. Qual Life Res. 2005 Apr;14(3):875-82. 